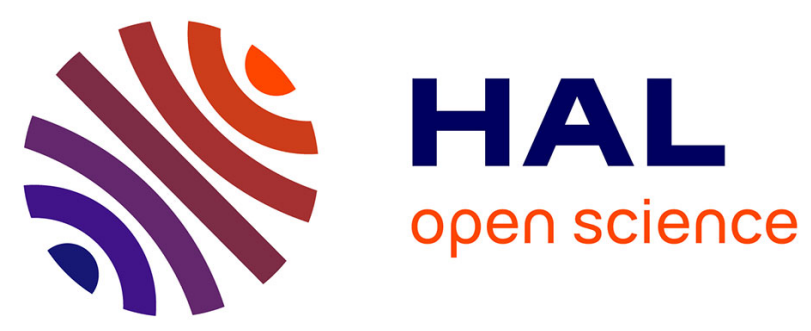

\title{
Highly sensitive measurements of the energy transferred during plasma sputter deposition of metals
}

\author{
L Bedra, a L Thomann, N Semmar, R Dussart, J Mathias
}

\section{To cite this version:}

L Bedra, a L Thomann, N Semmar, R Dussart, J Mathias. Highly sensitive measurements of the energy transferred during plasma sputter deposition of metals. Journal of Physics D: Applied Physics, 2010, 43 (6), pp.65202. 10.1088/0022-3727/43/6/065202 . hal-00569757

\section{HAL Id: hal-00569757 https://hal.science/hal-00569757}

Submitted on 25 Feb 2011

HAL is a multi-disciplinary open access archive for the deposit and dissemination of scientific research documents, whether they are published or not. The documents may come from teaching and research institutions in France or abroad, or from public or private research centers.
L'archive ouverte pluridisciplinaire HAL, est destinée au dépôt et à la diffusion de documents scientifiques de niveau recherche, publiés ou non, émanant des établissements d'enseignement et de recherche français ou étrangers, des laboratoires publics ou privés. 


\title{
Highly Sensitive measurements of the energy transferred during plasma sputter deposition of metals
}

\author{
L. Bedra, A.L. Thomann, N. Semmar, R. Dussart, J. Mathias \\ GREMI CNRS Polytech'Orleans, 14 rue d'Issoudun, BP6744, 45067Orleans cedex2, \\ FRANCE
}

\begin{abstract}
This work reports results obtained from heat flux measurements performed during the deposition of metallic thin films by low-pressure plasma sputtering. It introduces a sensitive diagnostic, which allows to perform such measurements directly during the process and to follow in real-time mechanisms involved in plasma/surface interaction. Although quantitative results are provided and discussed, the main scope of this article is a qualitative study of the sputter-deposition process via the energy flux transfers. The diagnostic developed for energy flux measurements is presented and the versatility of the experimental apparatus is described. Results on the study of the deposition of $\mathrm{Pt}$ (and Fe) thin films demonstrate a good reproducibility of the measurements and the ability to separate the energetic contributions of the main plasma $\left(\sim 300 \mathrm{~mW} / \mathrm{cm}^{2}\right)$ from the deposition process ones $\left(2\right.$ to $\left.23 \mathrm{~mW} / \mathrm{cm}^{2}\right)$. The influence of gas pressure, plasma power and target bias voltage, on the energy transferred to the silicon substrate is also studied.
\end{abstract}

\section{Introduction}

At present time, plasma enhanced processes, such as thin film deposition, etching, polymer functionnalisation, etc. are widely used for various industrial applications (microelectronics, protective coatings, material treatments...). To achieve the control of the process and help to the scale-up step, a better knowledge of the involved mechanisms is still necessary. Simultaneously to the plasma characterization, a good knowledge of the energy released onto the substrate is of crucial importance to control accurately the properties of the plasma modified material. Up to date, the fundamentals of these energy transfers are still a 
challenging research area [1]. The complexity of the energy transfer study is due to the fact that energetic contributions are of different kinds [2,3]:

- energy transfer by collision from electrons, ions, excited neutrals, sputtered atoms,

release of potential energy from metastable atoms, neutralization of ions or adatom condensation

- $\quad$ radiation from emitting plasma and heated devices (target, reactor walls etc.)

Depending on the plasma source, the reactor geometry, the substrate bias, the main species contributing to the energy transfer are different. Whereas charged particles are the main source of energy flux in case of biased substrates [4], contribution of electrons or neutrals may become significant in other cases [2-6]. Events occurring during plasma/surface interaction could be more complicated when chemical reactions (molecule dissociation, chemical etching, etc...) occur on the substrate surface [2,7]. Nevertheless, for non reactive sputtering, it is commonly admitted that the total energy transfer is composed of the contributions listed below [2,3]:

Sputter-deposition stage

- The adsorption enthalpy of the incoming atoms, usually referenced as condensation energy

- The kinetic energy of the sputtered atoms

- The energy of the gas ions, which recombine with electrons when colliding with the negatively biased target, becoming fast neutrals. Their key role has been evidenced in magnetron sputtering [6,8-11]

Plasma contribution

- The kinetic energy of gas atoms [12]

- The potential energy of metastables that can be partially released at the surface

- The kinetic energy of the charged particles: ions accelerated through the plasma sheath, and electrons which may interact with the substrate (eg in RF plasmas). For ions that are neutralized at the surface, the ionization energy has to be taken into account

Other transfer processes

- The radiative energy coming from both excited particles of the plasma and heated devices (vessel walls, metallic target ...) 
To distinguish each of these energy flux contributions, specific experiments have to be made in different configurations. Results provided by literature most often come from calculations based on temperature measurements $[4,12]$. Even if some sophisticated thermal probes have been developed [13-15], the total energy flux is always estimated a posteriori from thermograms recorded during the heating and cooling steps. Mathematical treatments are then employed to estimate the heat flux, that introduce systematic deviations. Moreover, with this kind of methods, it is not possible to evidence transfer mechanisms of different kinetics such as transfer by collision (instantaneous) or transfer involving a heating step (IR emission). Moreover, detection of transient or small energetic contributions (several $\mathrm{mW} / \mathrm{cm}^{2}$ ) could not be reasonably achieved.

The present work describes a study of the energy transfers onto a silicon substrate during deposition of metallic thin films by plasma sputtering. It is based on measurements performed with an energy flux diagnostic whose time resolution (1ms) is sufficient to follow the energy transfer occurring at the substrate surface in real time. Unlike Ellmer et al. [16] whose calorimetric sensor exhibits similar characteristics (i.e. sensitivity $\approx 20 \mu \mathrm{V} / \mathrm{mW} / \mathrm{cm}^{2}$ ), in the present energy flux diagnostic, the sensor is maintained at fixed temperature. This is favourable to repetitive heat loading and long operating time. Especially remarkable is the time rise, that is lower compared to the calorimetric sensor one (few ms). The experimental configuration has been chosen in order to separate heat fluxes coming from the argon plasma itself, and the ones due to the Sputter-Deposition Process SDP. An Inductively Coupled Plasma (ICP) reactor is used, where a metallic target can be independently DC biased. After a brief description of the experimental setup, results obtained with respect to experimental parameters are presented and discussed.

\section{Experimental apparatus}

The energy flux diagnostic is based on a commercial sensor built by Vatell Corporation [17] and composed of 1600 thermocouples $/ \mathrm{cm}^{2}$ mounted in series, and having a 6 mm diameter active surface (See Fig. 1a). Its principle is based on the Seebeck effect (thin film thermopile). The Vatell probe has been preferred to other commercial heat flux sensors because of its good sensitivity and time resolution ( 1ms). This Heat Flux Microsensor (HFM) is inserted in a water-cooled copper chamber (Fig. 1a). Hence, the HFM is set at a sufficiently low temperature $(278 \mathrm{~K})$ to make the heat flux permanently oriented toward the 
probe. Moreover, the lower the HFM temperature, the more accurate the energy flux signal (better signal to noise ratio). Measurements are carried out with a Keithley ® Nanovoltmeter 2182, since the output HFM voltages are of the order of $\mu \mathrm{V}$ at the relatively low energy fluxes reached in the present conditions.

The plasma reactor is a $40 \mathrm{~cm}$ diameter and $20 \mathrm{~cm}$ height chamber, in which pressure can be set down to $10^{-5} \mathrm{~Pa}$. The argon (Ar) gas inlet is set to $5 \mathrm{sccm}$ and the working pressure is adjusted between 0.5 and $5 \mathrm{~Pa}$. The experimental apparatus can be used in two configurations:

The first one is dedicated to the calibration procedure of the energy flux diagnostic. It has been widely described in previous paper [18]. For this step, a cylindrical (copper) homemade blackbody, surrounded by thermal shields, is inserted in the reactor at $0.5 \mathrm{~mm}$ from the HFM. The blackbody can be heated up to $500 \mathrm{~K}$ with a DC power supply. The calibration is performed in vacuum, so that only radiative transfers occur between the blackbody and the HFM, satisfying thus the NIST (National Institute of Standard and Technology) procedure [19].

The second configuration, designed for the SDP study, is schemed in figure $1 \mathrm{~b}$ ). The plasma is created in argon gas. A $13.56 \mathrm{MHz}$ generator is able to provide a power up to 1000 $\mathrm{W}$, via a matching box (reflected power $<5 \mathrm{~W}$ ). The sputtering target and the energy flux diagnostic are placed in a TCP reactor, $10 \mathrm{~cm}$ away from each other.The sputtered target is a $5 \times 5 \mathrm{~cm}^{2}$ and $0.5 \mathrm{~mm}$ thick metallic plate ( $\mathrm{Pt}$ or $\mathrm{Fe}$ ). A DC generator is connected to the target. Hence, the target voltage can be varied from -50 to $-350 \mathrm{~V}$. The substrate is a $0.5 \mathrm{~mm}$ thick and $1 \times 1 \mathrm{~cm}^{2}$ silicon wafer directly stuck [20] on the HFM active surface with a thermal paste, in order to reduce the thermal contact resistance. During the SDP, substrates are at floating potential.

\section{Results and discussion}

Previous studies, based on comparisons between experiments and COMSOL multiphysics simulations, have shown that the major part of the heat flux reaching the substrate is transferred to the HFM with negligible losses [21]. Since the HFM is water-cooled at $278 \mathrm{~K}$ and the substrates relatively thin, the heat flux remains directed toward the HFM. Therefore, the energy flux density on the substrate is deduced from the HFM measurement by multiplying the value obtained from the calibration curve with the ratio between the areas of the substrate and the HFM active surface. 


\section{1. SDP contribution to the global energy transfer}

A typical energy flux time evolution recorded during sputtering of $\mathrm{Pt}$ is presented in Fig. 2a. This experiment consists of a sequence of seven sputter-deposition steps, lasting 1 min each, with increasing negative bias voltages of the target. First of all, it is interesting to note that the background signal (heat flux density before switching on the plasma, i.e. for $\mathrm{t}<$ $1500 \mathrm{~s}$ ) is not equal to zero, but of the order of $18 \mathrm{~mW} / \mathrm{cm}^{2}$. This is due to radiative transfer between the reactor at room temperature and the substrate which is cooled down to $5^{\circ} \mathrm{C}$. After switching off the plasma $(\mathrm{t} \approx 3700 \mathrm{~s})$, the signal reaches again this value.

As soon as the plasma is turned on $(\mathrm{t} \approx 1500 \mathrm{~s})$, the heat flux through the substrate

surface increases sharply within $2 \mathrm{~s}$. The plasma contribution $\left(\Delta \varphi_{\text {plasma }}=300 \mathrm{~mW} / \mathrm{cm}^{2}\right)$ has been studied in [18] and is due to energy transfer from charged particles, especially Ar ions. After this switching on step, the signal continues on increase until it reaches a steady state (at about $2500 \mathrm{~s}$ ). This behaviour is attributed to the progressive heating of the reactor, inducing radiative transfer from the walls toward the substrate. This thermal contribution is detected by the HFM in addition to the plasma one.

From $t=2500 \mathrm{~s}$, small signals are visible on Fig. $2 \mathrm{~b}$, corresponding to the $1 \mathrm{~min}$ lasting sputter-deposition steps. One can first observe on each signal a sharp rise followed by three kinds of evolution:

the top of the first signals (-50 and $-100 \mathrm{~V})$ slowly increases, the last ones (from -200 V) decrease rather linearly, for $-150 \mathrm{~V}$ signal, a steady state is reached.

The slow rise is due to a radiative transfer from a heated device. As soon as the target is biased, it undergoes bombardment from the positive Ar ions. Its temperature rises (the target is not cooled) and it starts emitting in the infrared range. This radiation is detected by the HFM and partly overlaps the sputter-deposition signal.

To sustain this explanation, an experiment has been made with a $5 \times 5 \mathrm{~cm}^{2}$ piece of $\mathrm{Si}$ inserted between the sputtering target and the HFM. The Si shield was set very close $(0.5$ $\mathrm{mm}$ ) to the substrate-HFM system, in order to avoid any metal atom deposition on the silicon substrate. Silicon was chosen as a shield for its wide spectral transmittance (1-9 $\mu \mathrm{m})$ [22], which allows most of the IR radiation emitted by the target to reach the HFM. Note that the Si shield, left floating, can also be slightly heated under bombardment of low energy (e(Vp-Vf)) 
argon ions. When biasing the target in such a configuration, a small signal with a different shape from the one of SDP steps is observed (see Fig. 3). As a matter of fact, the sharp rise attributed to the atom deposition onto the substrate (that can be assisted by fast argon neutrals) does not exist anymore. However, as it can be seen in Fig. 3, there is a slow rise of the signal, which is attributed to a heating process of both sputtered target and Si shield. This experiment was thus useful to demonstrate that the sharp rise of the sputtering signal is due to collision processes (momentum transfer from particles) that we are interested in. The direct link between the target heating and the further evolution of the first SDP signals in the sequence is also checked. After a while, the target temperature becomes constant, and the SDP signal reaches a steady state as observed on Fig. 3 for $-150 \mathrm{~V}$.

The decreasing trend of the SDP signal (from $-200 \mathrm{~V}$ ) observed on Fig. $2 \mathrm{~b}$ can also be explained. This behaviour is attributed to the progressive coating of the upper reactor window just below the antenna by sputtered Pt atoms. This statement is supported by the fact that the signal only decreases during the sputter-deposition steps, whereas it is constant in-between (plasma contribution in Fig. 2b). If such a thin Pt layer covers the window, plasma-antenna coupling becomes less efficient (confirmed through the plasma colour change) and the electron density in the plasma is globally lowered. Consequently, the sputtering ions at the target are less numerous and the sputtering process becomes less efficient. This leads to a decrease of the energy transferred during the SDP. Moreover, after each sputtering step, the contamination layer being thicker, and the Ar ions density lower, the plasma contribution diminishes too.

From this first experiments it is seen that the contribution corresponding to the SDP $\Delta \varphi_{\text {sput }}$ is well quantifiable and strictly distinguished from the ICP plasma signal. It is proved that the HFM sensitivity is sufficiently high to detect the small amount of energy deposited by the SDP (from $2 \mathrm{~mW} / \mathrm{cm}^{2}$ to $23 \mathrm{~mW} / \mathrm{cm}^{2}$ ), hardly observed by classical thermal probes. It is also of particular importance to notice the ability of the HFM diagnostic to distinguish the heat flux transferred by fast mechanisms (conduction, adsorption) from those involving a thermal step (target heating,...).

Bias voltages ranging from -50 down to $-350 \mathrm{~V}$ were applied in order to study the effect of the target bias voltage on the energy transfer by the SDP onto the silicon substrate. At first glance, one can see on Fig. $2 b$ that the more negative the bias voltage, the higher the energy transfer. This will be discussed in the paragraph 3.3. 


\section{2. Reproducibility of heat flux measurements}

To investigate the reproducibility of both the SDP and the heat flux density measurements, additional experiments were carried out. The signal of a sequence of 6 sputterdeposition steps of Pt was recorded for a constant target bias voltage of -200 V (Fig. 4a and $4 \mathrm{~b})$. It is clearly shown that the corresponding heat flux density $\Delta \varphi_{\text {sput }}=8-9 \mathrm{~mW} / \mathrm{cm}^{2}$ remains constant from one step to another. Note that the heated target contribution was removed from the first one. This shows that the presence of the previously deposited metallic layers does not perturb the energy flux density measurements. Indeed, measurements being performed on a growing metallic layer containing some interfaces (sputtering off steps), an increasing thermal resistance should have been formed. This would have led to a decrease of the measured signal $\left(\Delta \varphi_{\text {plasma }}\right.$ and $\left.\Delta \varphi_{\text {sput }}\right)$. Possible perturbation of energy flux measurements induced by a change of the heat conduction were reported by Ellmer et al. [16] in the case of the calorimetric sensor they use. They conclude that for relatively low thicknesses of the deposited layer, this effect is negligible. The good reproducibility of the signal after each sputtering step indicates that measurements remain accurate enough during the growth of the metallic layer, in the experimental conditions used in Fig. 4.

Rather than the thickness, the interfaces created inside the layer during the plasma/surface interaction steps can influence the thermal conductivity. To check this point, we have performed two kinds of experiments where the growing regime of the Pt layer was modified.

First, we have compared a sequence of $6 \mathrm{Pt}$ sputtering steps of $1 \mathrm{~min}$ each with a continuous sputtering process of $6 \mathrm{~min}$; depositing respectively either 6 layers or one continuous layer for the same total sputtering time. Fig. 5 shows the HFM signal for the continuous sputtering step of $6 \mathrm{~min}$. Once again, one can see on this graph the effect of the processes described above: after the SDP contribution, a slow rise due to the radiative transfer from the heated target and a linear decrease, attributed to the growth of a Pt layer on the window. The value of $\Delta \varphi_{\text {sput }}=8-9 \mathrm{~mW} / \mathrm{cm}^{2}$ either calculated from the sharp rise or from the falling down of the signal, is the same for both cases: short time sputtering steps and long time continuous coating. The energy measurement is thus not affected by the layer growth mechanism.

The second conducted test consists of making several Pt sputtering steps (with increasing negative bias voltage), but by turning off the plasma between each. As shown in Fig. 6, the sputter-deposition contribution $\Delta \varphi_{\text {sput }}$ for a given target bias are the same on both 
experiments $\left(8-9 \mathrm{~mW} / \mathrm{cm}^{2}\right)$. This validates the rather accurate reproducibility of the diagnostic.

Thus, it is proved from these last experiments that, in the present conditions, the presence of a growing metallic layer on the substrate does not affect the energy flux measurements.

A comparison is made with the SDP of Fe in the same conditions than in Fig. 4 for Pt (see

Fig. $7 \mathrm{a}$ and $7 \mathrm{~b}$ ). The contribution of the $\operatorname{SDP} \Delta \varphi_{\text {sput }}=6-7 \mathrm{~mW} / \mathrm{cm}^{2}$ is well reproducible step to step. However, in this case, the plasma global signal does not decrease, even after several sputtering steps. This is also visible on the top of the sputtering slots, which never decreases. In opposite, they keep growing in a slow way due to the target IR emission and reach a steady state regime when the temperature becomes constant. The different behaviour observed on the sequence of 6 sputter-deposition steps between Pt and Fe is also a check of the reason for the decreasing tops observed on the Pt sputtering signals. The iron sputtering yield for $300 \mathrm{eV}$ argon ions is lower (0.6) than the platinum one (0.8) [23], thus the dielectric window under the excitation antenna is slower contaminated in the case of iron. The global Fe quantity deposited (in conditions of Fig. 5) after $6 \mathrm{~min}$ total sputtering time is not enough to significantly perturb the RF power/plasma coupling.

\section{3. Energy transfer during DSP versus experimental parameters}

All these experiments have shown that the diagnostic is suitable to study plasma/surface interaction via energy transfers, and for deposition process control. The order of magnitude of the global energy deposited onto the substrate is in good agreement with works on magnetron sputtering reported in literature $[2,5,16]$. An important result is that, according to the experimental configuration and to the sensitivity of the sensor, it is possible to separate the sputter-deposition contribution from those due to the plasma or from the residual heating of the reactor parts. This is an advantage compared to conventional techniques used for energy flux measurements during deposition. It is shown that in the present conditions, the energy part brought by condensing atoms and reflected neutrals is very small $(\approx 5 \%)$ compared to the contribution of the argon plasma. This is in correspondence with S. Mahieu et al. work [6,11], where the contributions to the energy momentum flux are calculated from plasma characterizations and simulations.

In the following, the diagnostic is used to study the influence of experimental parameters onto the SDP contribution: bias voltage, gas pressure and plasma power. 
Fig. 8 shows the linear evolution of the energy brought by the SDP with respect to the Pt target bias voltage. Obviously, as the target voltage becomes more negative, the kinetic energy and the flux of the $\mathrm{Ar}^{+}$attracted by the target increases. This leads to a more efficient sputtering process (higher sputtering yield of metal atoms). The metal atoms sputtered out of the target are thus more numerous. It has been shown that, the mean kinetic energy of sputtered atoms only weakly depends on the energy of the incoming argon ions $[9,24,25]$. The increase of the deposition contribution is thus mainly due to a rise of the number of condensing atoms. In the same manner, the contribution of the argon ions reflected by the target gets higher. This can be predicted from simple calculations given for example in [9]. Both phenomena tend to increase the global energy transfer coming from the SDP. The behaviour observed in Fig. 8 was thus expected.

For the most negative voltage, i.e. $-350 \mathrm{~V}$, the energy transferred to the Si substrate by the SDP is around $25 \mathrm{~mW} \cdot \mathrm{cm}^{-2}$. The target voltage corresponding to $\Delta \varphi_{\text {sput }}=0$ is about $25 \mathrm{~V}$. Taking into account the plasma potential ( $\approx 10 \mathrm{~V}$ in the present conditions), the corresponding energy of the sputtering argon ions can be estimated to $35 \mathrm{eV}$. It is interesting to compare this value with the sputtering threshold of Pt by Ar+ given in literature (25-30 eV) [23]. The good agreement between both values indicates that the energy transfer from metal sputtered atoms is the main contribution in the SDP.

Measurements performed at various gas pressures are presented in Fig. 9a. They have been made at 3 different bias voltages (-100, -200 and $-350 \mathrm{~V})$ and for each of the 4 pressure values $(0.5,1,3$ and $5 \mathrm{~Pa})$. As expected, the energy transferred by the plasma increases with pressure. This is because the ion density increases when the plasma gets more confined by collisions. This point is reported in [26] for instance.

Since the number of collisions of plasma particles increases with pressure, the sputtered atoms are also subjected to deviations: they are scattered away from their path to the substrate. Moreover, they lose a significant part of their kinetic energy [3,9,24,27]. Consequently the energy contribution of the condensed atoms (or land reflected neutrals) $\Delta \varphi_{\text {sput }}$ drops down (see Fig. 9b).

One can notice on Fig. 9a that the lower the pressure, the better resolved the sputtering slots. Zooms of two sputtering steps for $-200 \mathrm{~V}, 0.5 \mathrm{~Pa}$ and $3 \mathrm{~Pa}$ respectively are presented in Fig. 10. It is clearly seen that the deposition signal shape changes with the pressure. Therefore, mechanisms involved in the energy transfer are varying with pressure. It has been 
reported in literature that the gas temperature increases with the pressure [28]. This is evidenced on Fig. 10b where the gas thermal contribution dominates the signal.

In Fig. 11, the energy flux density evolution is plotted versus the plasma power. The plasma is switched 'on' and 'off' before and after each power value. As expected, the evolution of the plasma energy contribution is proportional to the RF power. Since the electron density linearly evolves with power in an ICP plasma, the energy transferred to the surface increases. A good agreement has been found between ionic current measured with Langmuir probe (not given here) and plasma contribution to the global heat flux transfer. This suggests that the energy transferred by the plasma as a function of the power mainly depends on the ions flux evolution. This is confirmed by estimations performed in a previous work [18] in similar experimental conditions (ICP argon plasma, 0.5 $\mathrm{Pa}$, substrate at floating potential). The ion energetic contribution was estimated to about $80 \%$ of the total.

As RF power increases, the energy transfer due to the SDP rises (Fig. 11b). This is due to a higher ion density in the plasma leading to an enhanced sputtering process. The number of adsorbed atoms onto the substrate becomes more significant, increasing the global energy flux transferred to the substrate.

\section{Conclusion}

This work highlights application of a sensitive energy flux sensor to a semiquantitative investigation of the energy transfers occurring during SDP. Direct energy flux measurements are performed with an original diagnostic developed at the laboratory. Results show that such a system allows to follow the real time evolution of the energy flux transferred to the substrate. From experiments presented here, the ability of the diagnostic to monitor a deposition process is clearly established. According to the chosen experimental conditions and to the sensor sensitivity, the contribution of the SDP was successfully isolated from the Ar plasma one. Moreover, the short response time allows to get rid of the slow contributions due to IR radiations emitted from the heated parts of the chamber.

The influence of several experimental parameters (Ar pressure, target voltage and RF power) on the energy transferred to the substrate was studied. It is shown that plasma contribution increases with respect to both pressure and RF power. These general trends, already reported in the literature, were expected. For the SDP contribution, a linear rise with bias voltage and plasma power, and a decrease as a function of the pressure was qualitatively 
evidenced. This was explained in terms of sputtered atom and reflected neutral kinetic energy and flux variations. Another paper is in progress where measured energy transfers from the SDP will be compared to estimations from energy balances. 


\section{Acknowledgements}

This work is supported by the ANR (Agence Nationale pour la Recherche). 


\section{References}

[1] Han J G 2009 J Appl D: Appl Phys 42043001

[2] Kersten H, Deutsch H, Steffen H, Kroesen G M W and Hippler R 2001 Vacuum 63385

[3] Ekpe S D and Dew S K 2008 in Reactive Sputter Deposition edited by Depla D and Mahieu S (Berlin Heidelberg: Springer series in materials science) 109

[4] Paturaud C, Farges G, Sainte Catherine M C, and Machet J 1998 Surf Coat Technol 98 1257

[5] Ekpe S D and Dew S K 2003 J Vac Sci Technol A 21(2) 476

[6] Mahieu S and Depla D, 2009 JAP 42053002

[7] Dussart R, Thomann A L, Pichon L E, Bedra L, Semmar N, Lefaucheux P, Mathias J, and Tessier Y 2008 Appl Phys Letters 93131502

[8] Coufal H, Winters H F, Bay H L, Eckstein W 1991 Phys. Rev. B 444747

[9] Drüsedau T P, Bock T, John T M, Klabunde F, Eckstein W 1999 J Vac Sci Technol A 17(5) 2896.

[10] Somekh R E 1984 J Vac Technol A 2(3) 1285

[11] Mahieu S, Van Aeken K, Depla D 2008 JAP 104113301

[12] Piejak R, Godyak V, Alexandrovich B, and Tishchenko N 1998 Plasma Sources Sci Technol 7590

[13] Thornton J A and Lamb J L 1984 Thin Solid Films 11987

[14] Kersten H, Rohde D, Steffen H, Hippler R, Swinkels G H P M, Kroesen G M W 2001 Appl. Phys A 72531

[15] Wolter M, Stahl M, Kersten H 2009 Vacuum 83768

[16] Ellmer K and Mientus R 1999 Surf Coat \& Technol 116-119 1102

[17] http:/www.vatell.com

[18] Thomann A L, Semmar N, Dussart R and Mathias J 2006 Review of Scientific Instruments 77 033501-1

[19] Murthy A V, Tsai B K, and Saunders R D 2000 J Res Natl Inst Stand Technol 105293.

[20] See http://www.electrolube.com/docs/bheat.html for information on the thermal paste.

[21] Bedra L, Semmar N, Thomann A L, Dussart R, Mathias J, Tessier y 2008 Proceedings

(CD) of the $5^{\text {th }}$ European Thermal-Sciences Conference (Eurotherm) Eindhoven

[22] Von Allmen 1987 Ion Beam Interactions with Materials (Springer Verlag)

[23] Yamamura Y and Tawara H 1996 Atomic Data and Nuclear Data Tables 62(2) 149

[24] Brault P, Thomann A L, Rozenbaum J P, Andreazza C, Andreazza P, Rousseau B et al, 2000 Recent Res Devel Vacuum Sci \& Tech 235 
[25] Wendt R, Ellmer K and Wiesemann 1997 J. Appl. Phys. 82(5) 2115

[26] Thomann A L, Charles C, Brault P, Laure C, Boswell R 1998 Plasma Sources Sci Technol 7245

[27] Mahieu S, Buyle G, Depla D, Heirwegh S, Ghekiere P, De Gryse R 2006 NIMB 243313

[28] Ekpe S D and Dew S K 2006 J. Phys. D: Appl. Phys. 391413 
Figure captions

Figure 1: a) Photos showing the heat flux micro-sensor (inch scale) and its integration in the water-cooled substrate holder, b) schematic of the ICP plasma reactor in sputter-deposition configuration.

Figure 2: a) Energy flux measurement during a sequence of seven sputter-deposition steps of $\mathrm{Pt}$, in $0.5 \mathrm{~Pa}$ pressure and $400 \mathrm{~W}$ argon plasma. b) Zoom of Fig. 2a showing the 1 min sputterdeposition steps performed with target voltages ranging from -50 to $-350 \mathrm{~V}$

Figure 3: Energy flux measurement during sputter-deposition step performed with a Si shield located in front of the substrate. In this configuration no particles coming from the SDP are able to reach the surface. The recorded signal is thus only due to the contribution from heated devices (metal target and Si shield).

Figure 4: Test of reproducibility for Pt deposition at $-200 \mathrm{~V}$ bias target, in $0.5 \mathrm{~Pa}$ and $400 \mathrm{~W}$ argon plasma. a) Energy flux measurements, b) zoom of Fig. 4a showing the 1 min sputterdeposition steps performed with a target voltages of $-200 \mathrm{~V}$. The SDP contribution is the same for each sputter-deposition step.

Figure 5: Energy transferred during a continuous 6 min sputter-deposition of $\mathrm{Pt}$ at $0.5 \mathrm{~Pa}$, $400 \mathrm{~W}$ and $-200 \mathrm{~V}$. The sharp rise is due to transfer from sputtered metal atoms and reflected neutrals. The following slow evolution (in the circle) is characteristic of radiative transfer from the heated target. The slow global decrease is attributed to the progressive coating of the upper window by Pt atoms, which influences the RF power/plasma coupling.

Figure 6: Comparison of the energy transfer during SDP of Pt when the plasma is turned off between each sputtering step or not, (0.5 Pa and 400W). The good agreement between both sets of values shows that the growing regime of the Pt layer on the substrate does not influence the measurements.

Figure 7: Test of reproducibility for Fe sputter-deposition at $-200 \mathrm{~V}$ bias target, in $0.5 \mathrm{~Pa}$ and $400 \mathrm{~W}$ argon plasma. a) Energy flux measurements, b) zoom of Fig. 7a showing the $1 \mathrm{~min}$ sputter-deposition steps performed with a target voltages of $-200 \mathrm{~V}$. As in the case of $\mathrm{Pt}$ deposition, the SDP contribution is the same for each sputter-deposition step. However, the shape of the sputter-deposition signals indicates that there is no modification of the RF power/plasma coupling (no decrease after several sputter-deposition steps).

Figure 8: Energy flux density due to the SDP of Pt versus the target bias voltage in a $0.5 \mathrm{~Pa}$ and $400 \mathrm{~W}$ argon plasma. The increase of the SDP contribution when the target bias voltage becomes more negative was expected, and is explained by a rise the flux and energy of the Ar 
sputtering ions at the target. The value at $\Delta \varphi_{\text {sput }}=0$ (in the circle) is close to the theoretical sputtering yield of $\mathrm{Pt}$ atoms by Ar ions. This suggests that the main part of the energy transferred during the SDP is carried by the sputtered metal atoms (against the reflected neutrals).

Figure 9: a) Energy flux measurements performed at four argon pressures, for $400 \mathrm{~W} R \mathrm{~F}$ power and $-100 \mathrm{~V},-200 \mathrm{~V},-350 \mathrm{~V}$ target bias voltages. b) Evolution of the SDP contributions versus pressure for -200 and $-350 \mathrm{~V}$ target bias voltages. The observed decrease is explained by the increase of the collision number between particles (sputtering ions, sputtered metal atoms, reflected neutrals) and argon atoms, which leads to scattering phenomena and reduction of the mean kinetic energy.

Figure 10: Shape of the sputter-deposition signals for two argon pressures at 400W RF power: a) $0.5 \mathrm{~Pa}$ and b) $3 \mathrm{~Pa}$. It is clearly seen that the argon pressure influences the SDP signal, and consequently the mechanisms of energy transfer. At $3 \mathrm{~Pa}$ a thermal contribution is visible together with the fast transfer from SDP particles.

Figure 11: Energy transfer as a function of the RF plasma power (1Pa argon pressure): a) heat flux measurements, b) SDP contribution. The energy transferred during the SDP increases with the RF power because of the global rise of the ionisation degree in the plasma. The number of sputtering ions at the target becoming higher, the sputtering process is enhanced. 
Figures

Fig.1-a

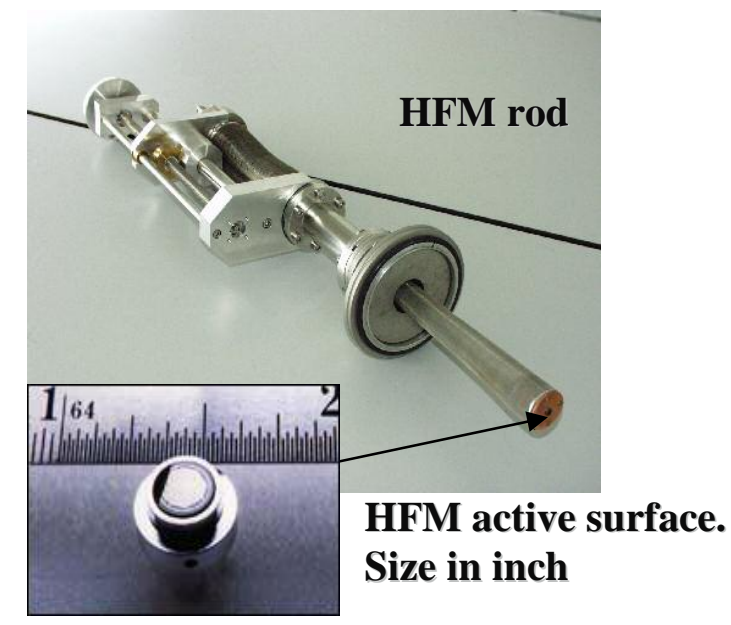

Fig.1-b

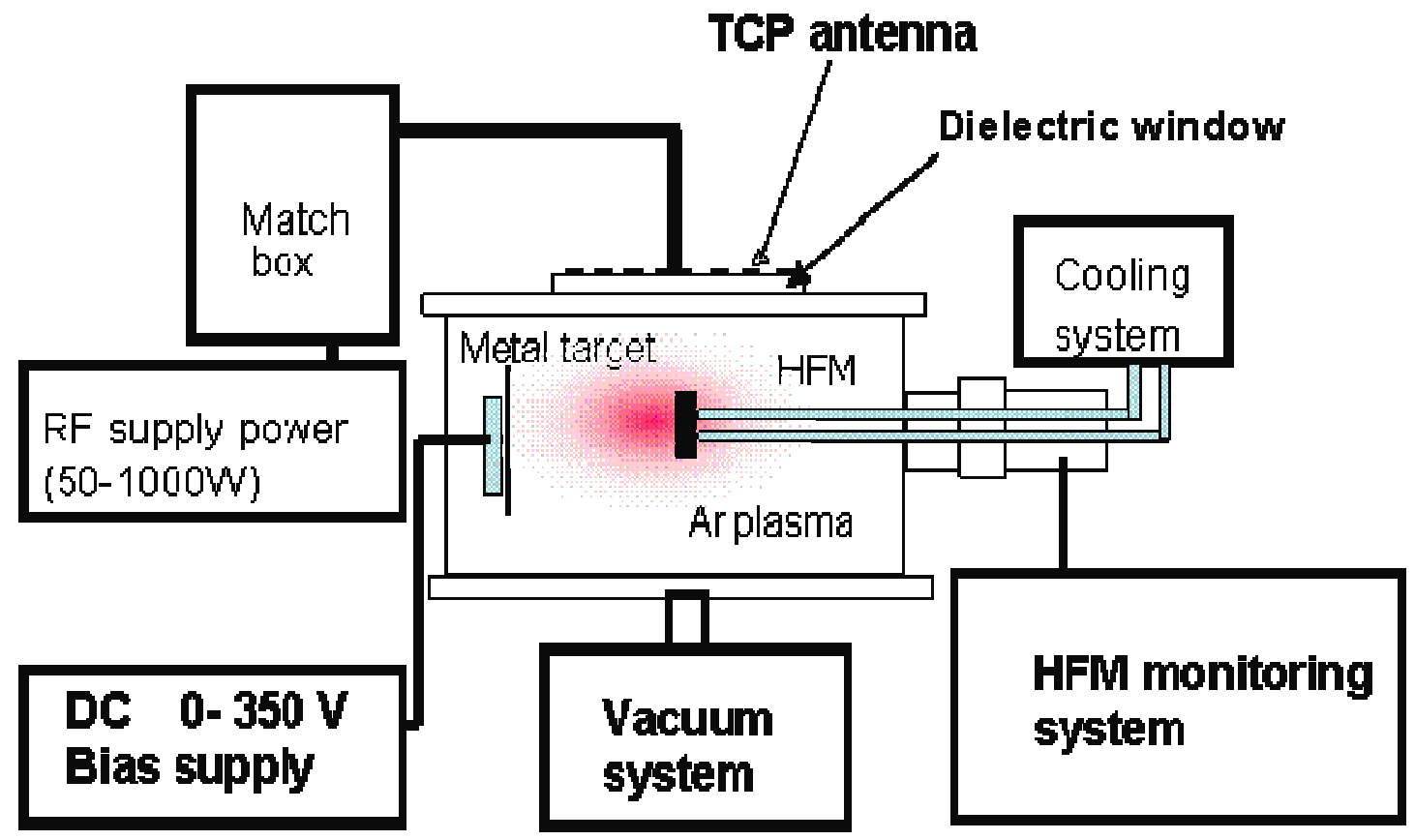


Fig.2-a

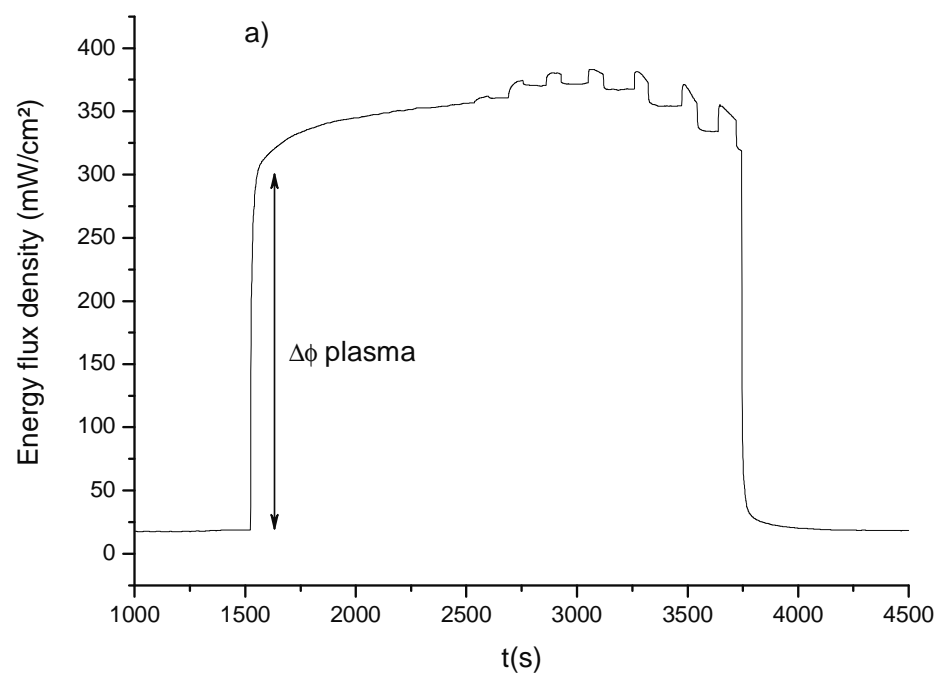

Fig.2-b

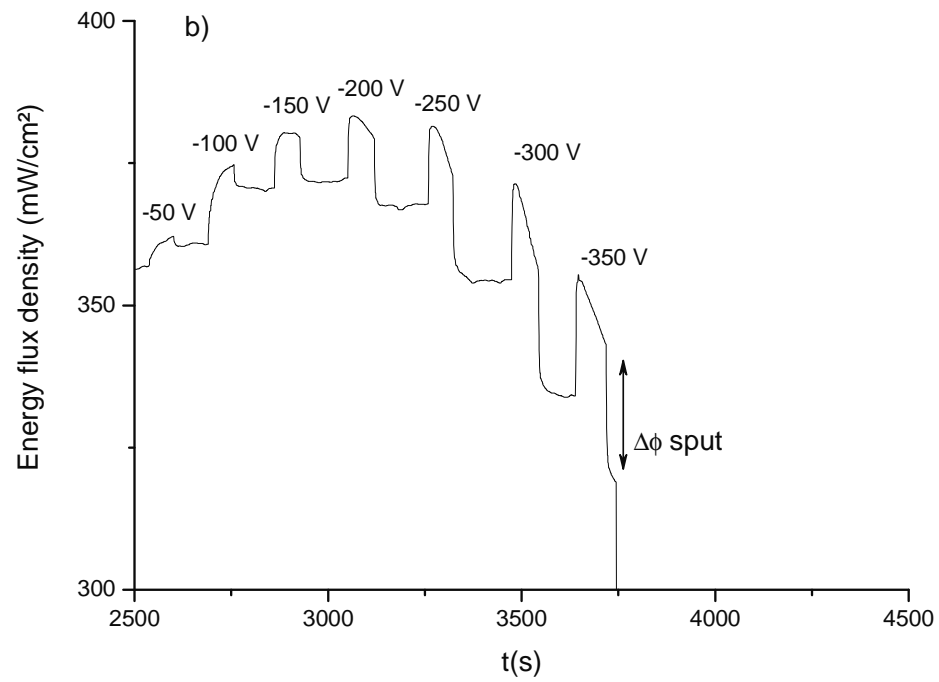


Fig.3

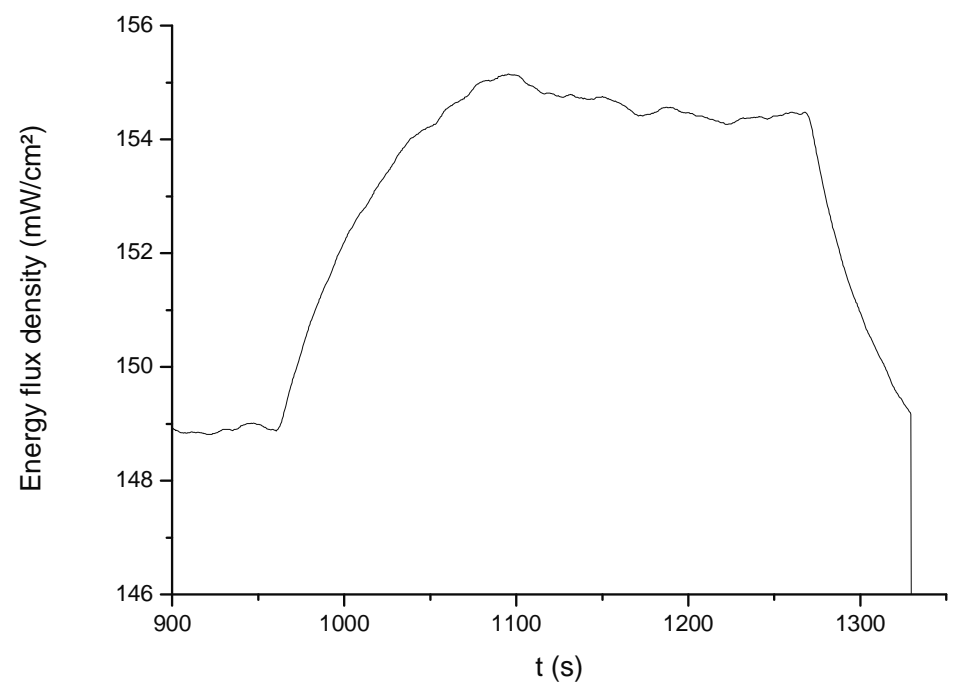


Fig. 4-a

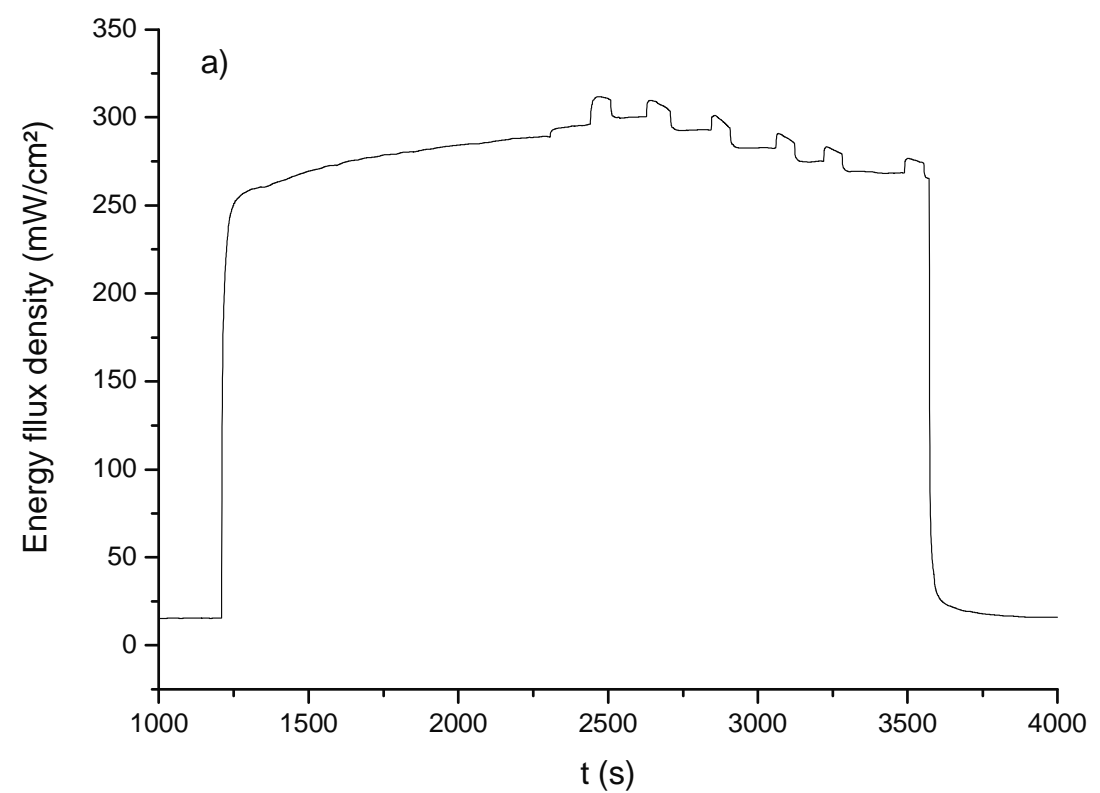

Fig. 4-b

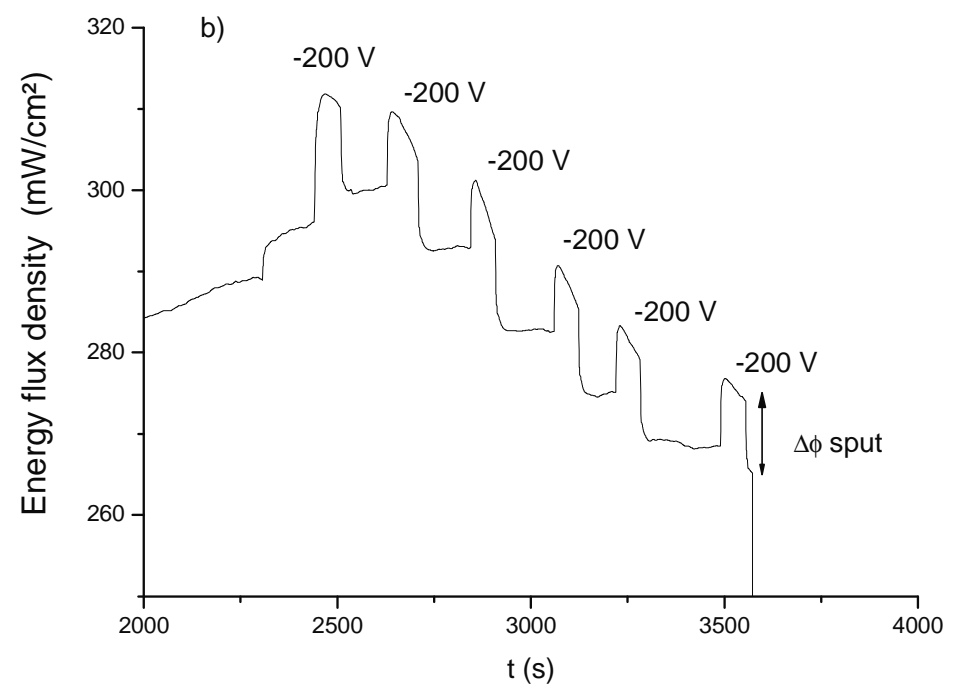


Fig. 5

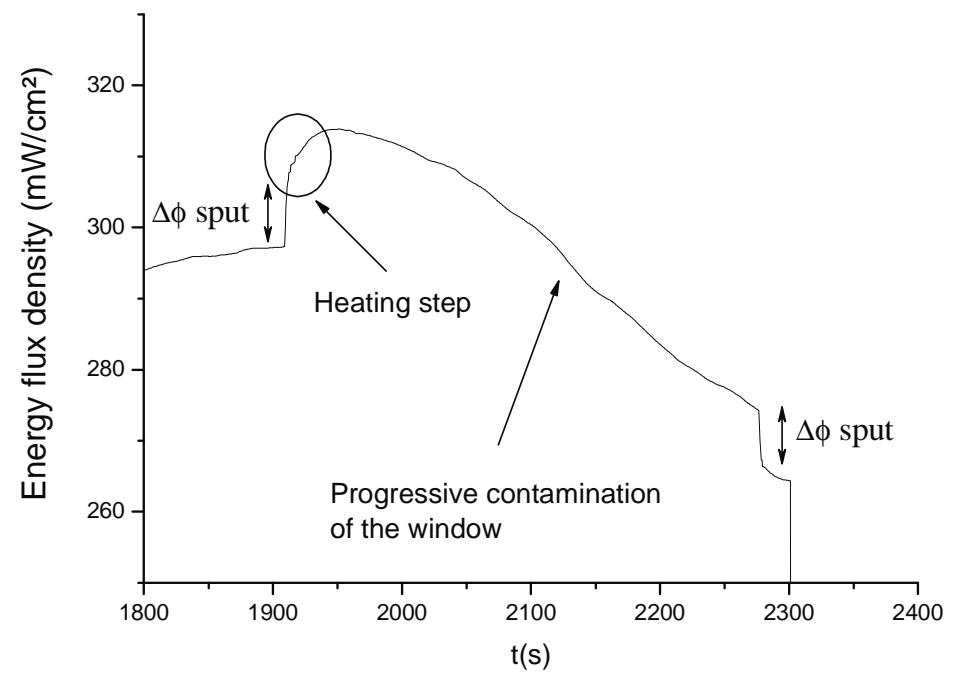


Fig. 6

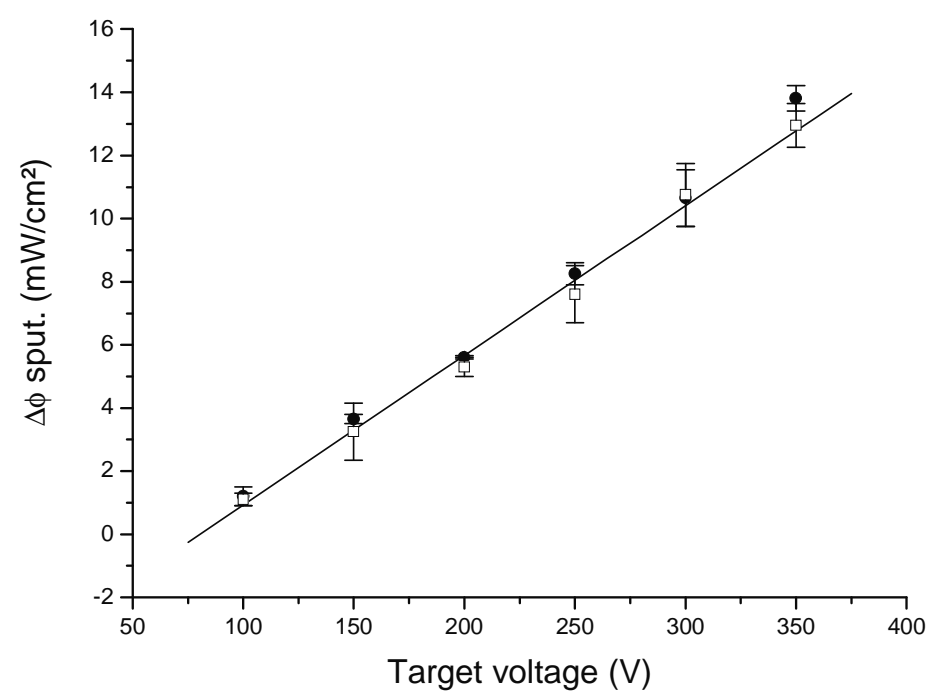


Fig. 7-a

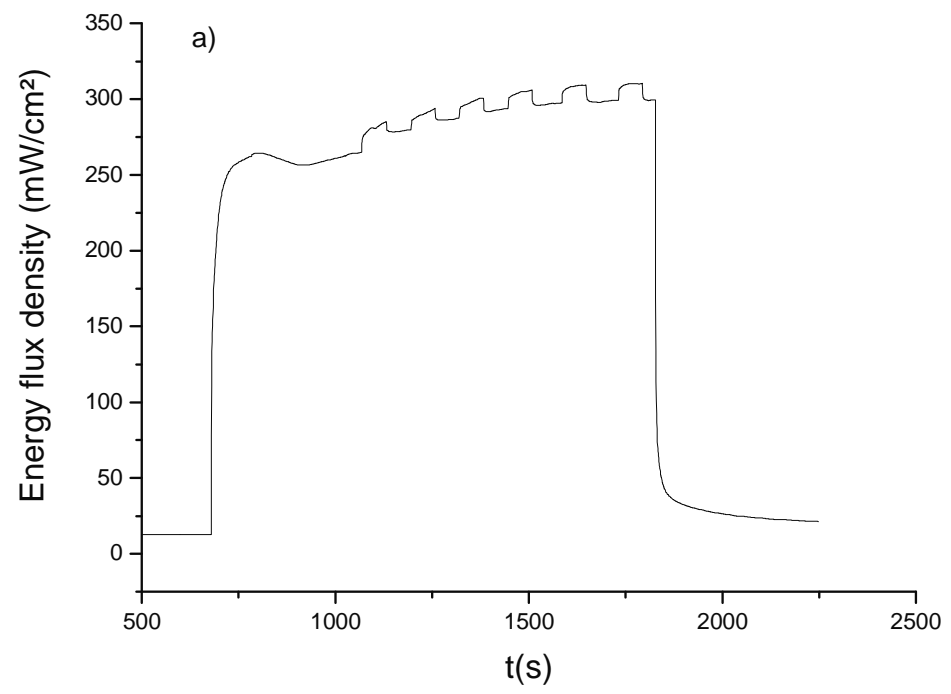

Fig. 7-b

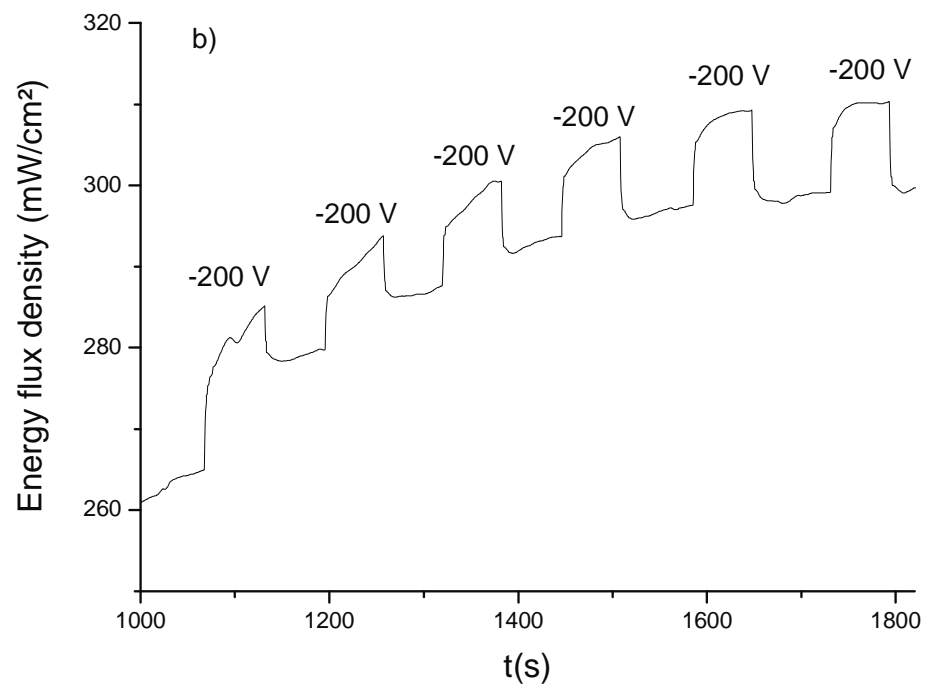


Fig.8

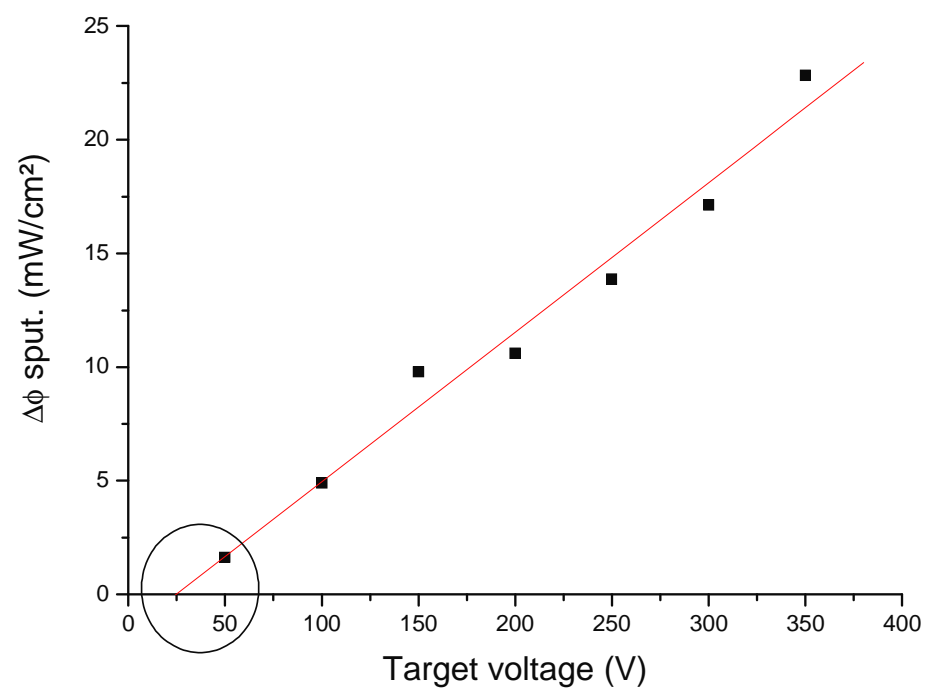


Fig. 9-a

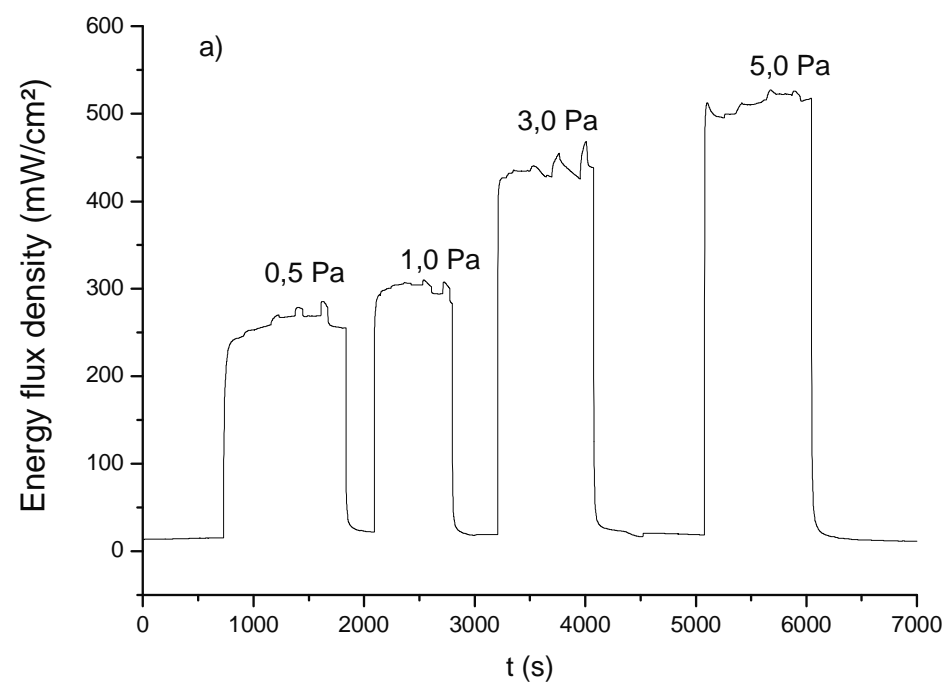

Fig.9-b

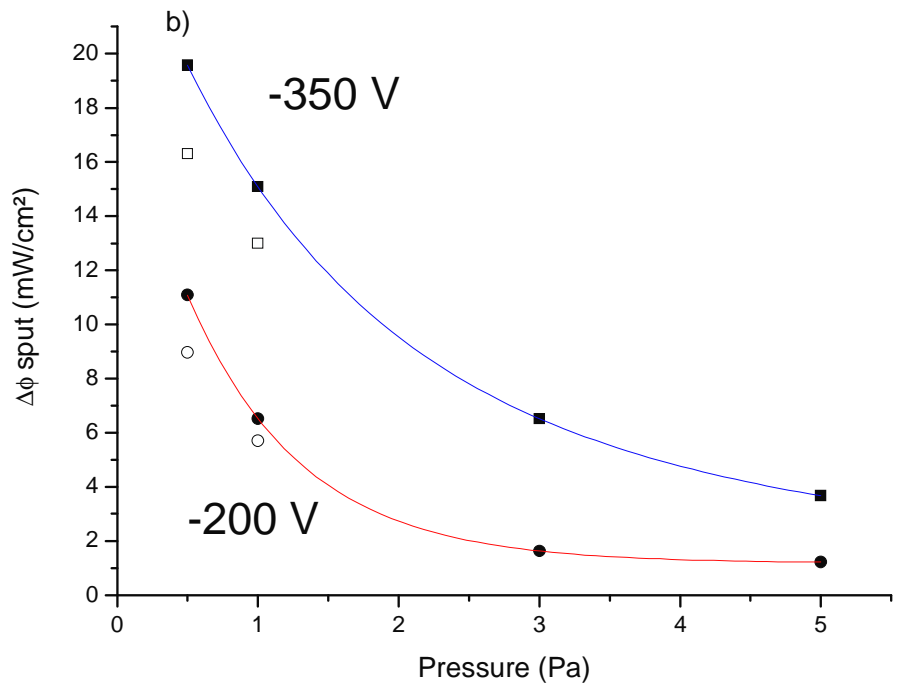


Fig. 10
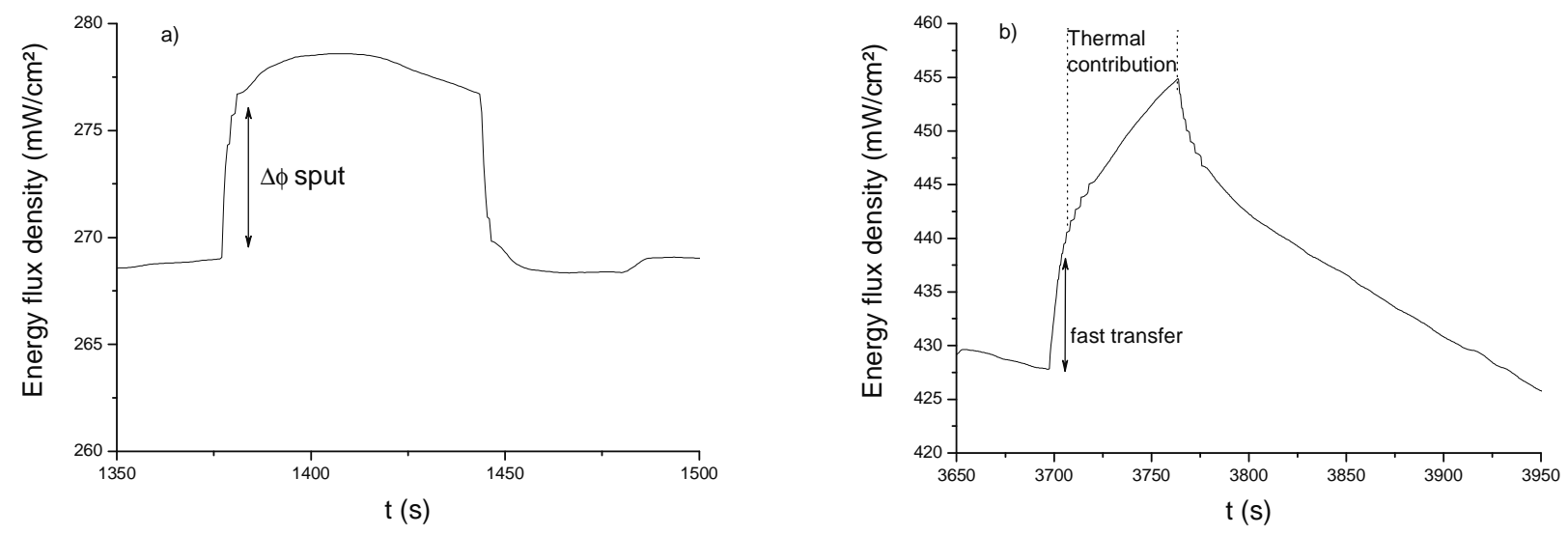
Fig. 11-a

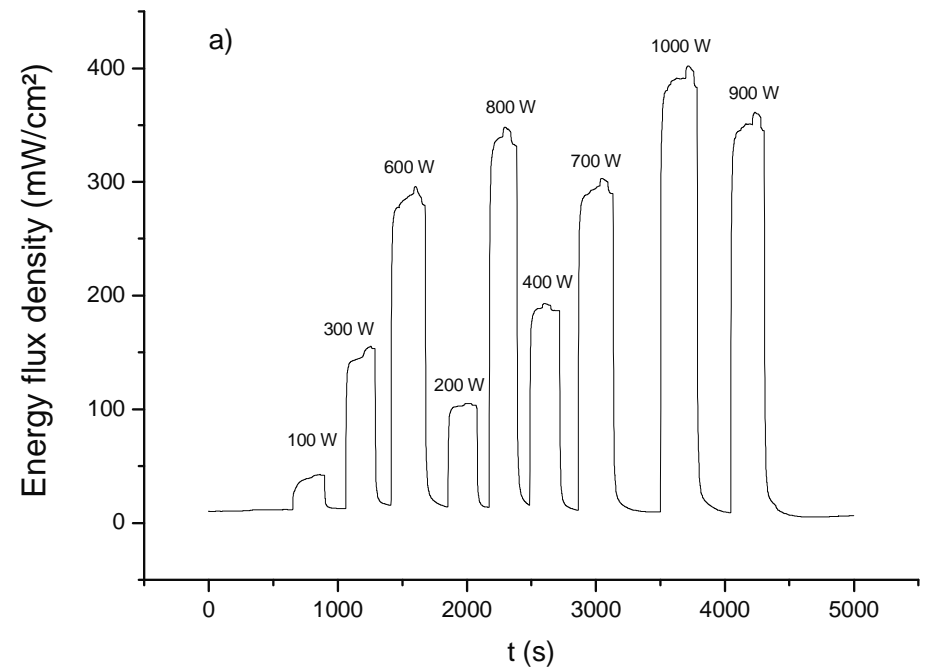

Fig. 11-b

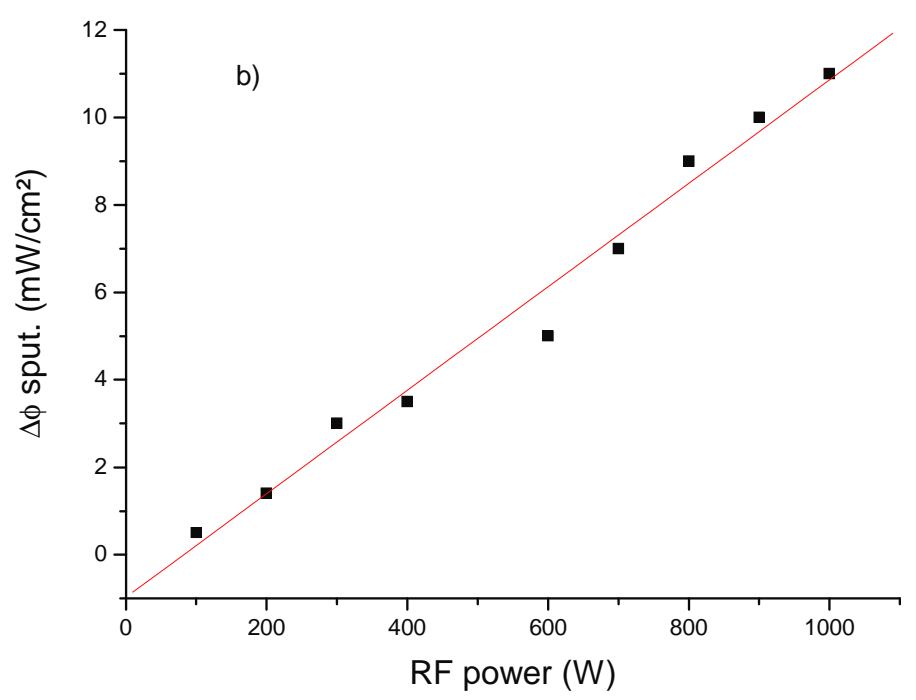

